DS (Child) H J 2019; 35(2) : 189

\title{
Postgraduate courses/training in paediatrics and child health
}

1. FCPS in paediatrics : Twice in a year, in the months of January and July.

2. Recognized center by BCPS for training in FCPS (Paeditric surgery) .

3. Recognized centre for course and training in different subspeciality as: Neonatology, Pediatric Nephrology, Paediatric Haematology and Onchology, Paediatric Pulmonology and Paediatric Neuroscience.

3. MD/MS in paediatrics : Part I: In the month of January every year; 2nd and 3rd parts twice every year.

4. DCH course : Once in a year in the month of July.

5. Three months certificate course : The institute every year runs 3 months certificate course on paediatrics for general practitioners \& other post graduate candidates e.g. MCPS.

(1st August - 31st October)

6. Training programme on IMCI (Integrated management of childhood illness), Essential Newborn Care for doctors and nurses, KMC (Kangaroo Mother Care) traing, ETAT (Emmergency Triage, Assessment and Treatment) training.

Contact Person : Prof. Md. Jahangir Alam

DCH, MCPS, FCPS (Paed)

Editor

Dhaka Shishu (Children) Hospital Journal

Bangladesh Institute of Child Health

Sher-e-Bangla Nagar, Dhaka- 1207.

Cell: 01716344008

Contact $\quad$ : Phone No. 9113048, 8122514, PABX: 9104211-20, Ext. 215

E-mail: infodshjournal@gmail.com, profdrmdjahangiralam@gmail.com 
DS (Child) H J 2019; 35(2) : 190

\section{Students Qualified from Bangladesh Institute of Child Health}

\section{Undergoing Courses of BICH}

\begin{tabular}{ll}
\hline Institution & Courses \\
\hline Bangabandhu Sheikh Mujib Medical University & MD (Pediatrics) \\
& MD Pediatrics Nephrology (sub-specialty) \\
& MD Neonatology (sub-specialty) \\
Bangladesh College of Physicians and Surgeons (BCPS) & DCH \\
& MS (Pediatrics Surgery) \\
& FCPS Part II (Paediatrics) \\
& FCPS Pediatric Nephrology \\
& FCPS Hematology \& Oncology \\
& FCPS Pediatric Surgery \\
& FCPS Pediatric Neurology \& Development \\
Dhaka University & FCPS Pediatric Pulmonology \\
Bangladesh Nursing Council & B.Sc in Health technology (Lab) \\
& Diploma in Pediatric Nursing
\end{tabular}

Student Qualified from BICH till JulyDecember 2019

\begin{tabular}{lc}
\hline Course & Number \\
\hline DCH & 370 \\
MD (Pediatrics) & 116 \\
MS (Pediatrics) & 109 \\
FCPS (Pediatrics) & 28 \\
MD (Neonatology) & 13 \\
MD (Pediatrics Nephrology) & 5 \\
\hline Total & $\mathbf{6 4 1}$ \\
\hline
\end{tabular}

Foreign Student Qualified from BICH till January 2013

\begin{tabular}{clc}
\hline Country of origin & Course & Number \\
\hline Nepal & DCH & 23 \\
& MS (Ped Surgery) & 2 \\
& MD (Ped) & 1 \\
India & MD (Ped) & 1 \\
Iran & DCH & 1 \\
Iraq & DCH & 1 \\
Somalia & DCH & 1 \\
Sudan & DCH & 1 \\
\hline Total & & $\mathbf{3 1}$ \\
\hline
\end{tabular}

Present Students (June 2019 to December 2019)

\begin{tabular}{lc}
\hline Name of Courses & Number of Students \\
\hline MD (Paediatrics) Phase - A & 25 \\
MD Neonate Phase - A & 06 \\
MD Paediatric Nephrology- Phase - A & 04 \\
MS (Paediatric Surgery) Phase - A & 19 \\
FCPS (Paediatric) - Part-II & 0 \\
MD (Paediatric) Part-III & 10 \\
MD (Paediatrics) Part-II & 0 \\
MS (Paediatric Surgery) Part-III & 19 \\
DCH (old) & 02 \\
DCH (New) & 10 \\
MD (Paediatrics) Phase-B & 32 \\
MD (Neonate) Phase-B & 06 \\
MD (Nephrology) Phase-B & 04 \\
MS (Paediatric Surgery) Phase-B & 20 \\
Total & 137
\end{tabular}


DS (Child) H J 2019; 35(2): 191

\section{Seminar/Symposium \& CME/CPD Programs held at BICH (July- December 2019)}

\begin{tabular}{llll}
\hline Sl. No. & Topic & Unit Date \\
\hline 01. & Inborn Errors of Metabolism & MU - III & 21.07 .2019 \\
02. & Hemolytic Uremic Syndrome & MU - II & 29.07 .2019 \\
03. & Management of Congenital Anomaly of Kidney & MU - II \& PNSB & 18.08 .2019 \\
04. & Dengue fever: Issues and controversies & MU - VI & 08.08 .2019 \\
05. & Movement Disorder in Children & MU - III & 25.08 .2019 \\
07 & Identification of Neonatal Shock \& Management & MU - IV & 22.09 .2019 \\
06. & Talipes Equinovarus (TEV): An Update & SU - I & 27.10 .2019 \\
07. & Respiratory sounds & MU - V & 24.11 .2019 \\
08. & Influenza : A Forgotten Pandemic & MU - VI & $29.12-2019$ \\
\hline
\end{tabular}

